\title{
Optimal patient classification via statistical decomposition of a 3D left-ventricular atlas
}

\author{
Pau Medrano-Gracia ${ }^{1 *}$, Brett R Cowan', David A Bluemke², J Paul Finn, Daniel C Lee³, Joao A Lima", \\ Avan Suinesiaputra', Alistair A Young ${ }^{1}$ \\ From 16th Annual SCMR Scientific Sessions \\ San Francisco, CA, USA. 31 January - 3 February 2013
}

\section{Background}

Statistical quantification and classification of heart disease, using clinical indices such as ejection fraction (EF) or leftventricular mass (LVM), are used routinely in clinical practice for both diagnosis and prognosis. However, 3D CMR of the left ventricle (LV) provides a wealth of shape features which can maximise the classification power and accuracy of such indices. To exploit these, we propose a framework whereby shape parameters of a 3D LV finite element atlas are used to optimally classify patients according to linear statistical decompositions (Fig. 1).

\section{Methods}

Finite-element shape models of the LV were customised to 600 cardiac MRI volumes with previously standardised and validated software (CIM v. 6.0, Auckland, NZ). The dataset comprised 300 community-based participants without a history of cardiovascular disease, aged 45-84 from 4 ethnic groups from the Multi-Ethnic Study of Atherosclerosis (MESA) cohort (1) and 300 patients with myocardial infarction from the Defibrillators To Reduce Risk By Magnetic Resonance Imaging Evaluation (DETERMINE) cohort (2) made available through the Cardiac Atlas Project (3). Bias due to scan protocol differences between cohorts was corrected (4). Shape classifiers were constructed to optimally detect which cohort each case belonged. A comparison between principal component analysis (PCA) and information preserving component analysis (IPCA) (5) was performed, using shapes at enddiastole (ED), end-systole (ES) and the difference in shape between ED and ES (ED-ES) which included information on regional wall motion. Traditional clinical classifiers of $\mathrm{EF}$, end-diastolic/end-systolic volume (EDV/ESV) and

${ }^{1}$ Auckland Bioengineering Institute, The University of Auckland, Auckland, New Zealand

Full list of author information is available at the end of the article
LVM were also included for comparison. Ten-fold crossvalidation experiments were performed in which $90 \%$ of the cases were used for training and $10 \%$ for validation, repeated 10 times with different training/validation cases.

\section{Results}

Classification results showed that this framework was able to determine clinically relevant modes and that IPCA achieved the lowest error rates using the ED-ES shape difference, with a single classifier number. This classifier can also be used to quantify severity of disease (degree of match with each group). Ten-fold cross-validation experiments corroborated the robustness of this approach which averaged $100 \%$ specificity and $99 \%$ sensitivity for IPCA versus $83 \%$ and $93 \%$ respectively when compared to ejection fraction (Table 1). Further, by back-projecting the optimal classifier onto the atlas, we were able to quantify and visualise which regions of the LV had the most weight in the decision. For the ED-ES classifier, this was a combination of volume and wall thickness change.

\section{Conclusions}

This work shows the potential of shape based classification in the automated identification and quantification of heart disease.

\section{Funding}

This work was supported by award no. R01HL087773 from the NHLBI. The content is solely the responsibility of the authors. The NIH (5R01HL091069) and St. Jude Medical provided grant support for the DETERMINE trial. MESA was supported by contracts N01-HC-95159 through N01-HC-95169 from the NHLBI and by grants UL1-RR-024156 and UL1-RR-025005 from NCRR. 


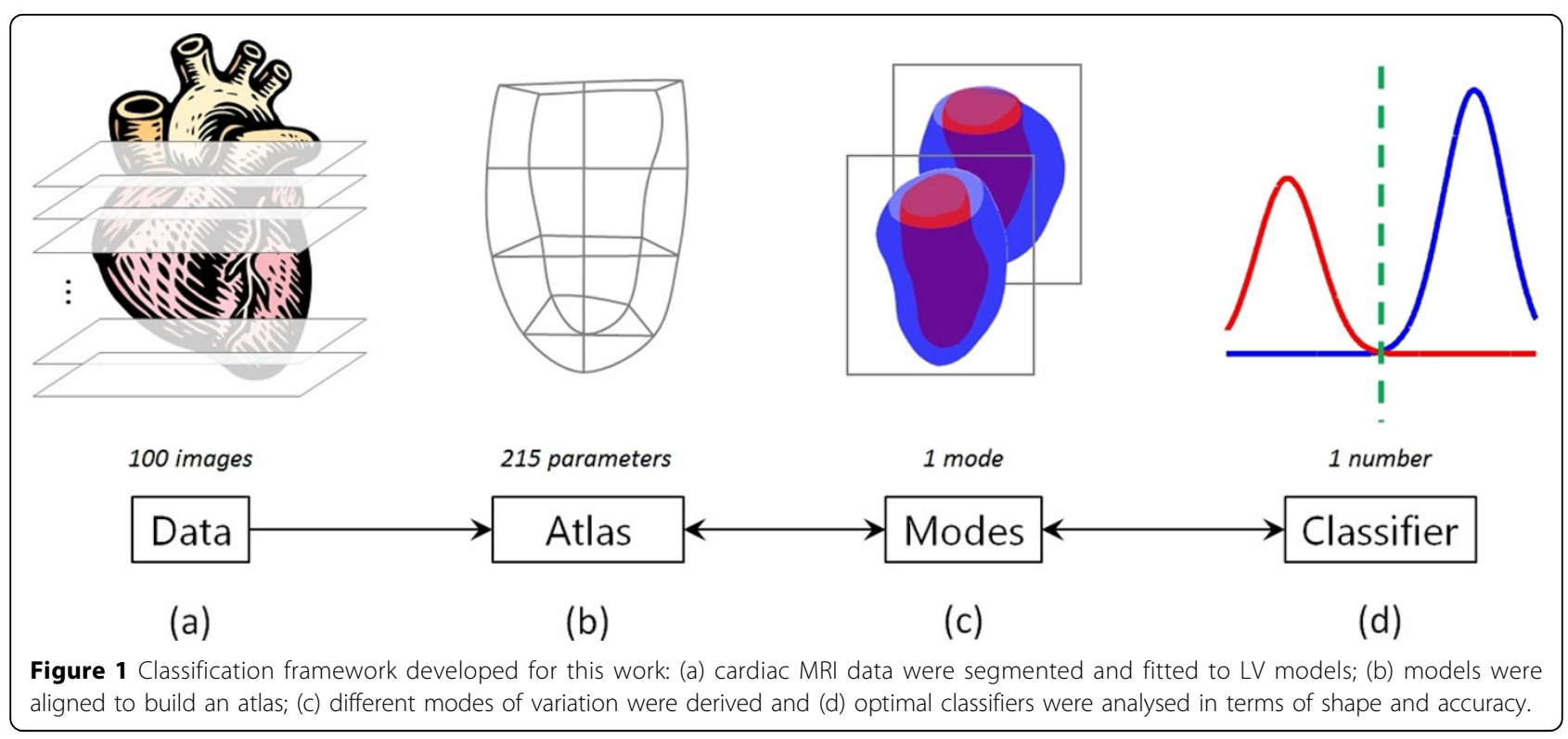

Table 1 Specificity and sensitivity are shown in brackets (in that order) for the cross-validation experiments.

\begin{tabular}{cccc}
\hline & ED & ES & ED-ES \\
\hline PCA & $(53.8,54.4)$ & $(77.5,75.6)$ & $(89.4,87.9)$ \\
IPCA & $(96.6,91.2)$ & $(96.2,98.0)$ & $(100,99.0)$ \\
\hline EF & & & $(82.7,93.2)$ \\
EDV & $(81.6,92.4)$ & $(79.2,98.3)$ & \\
ESV & $(67.1,70.1)$ & & \\
LVM & $(1)$ & \\
\hline
\end{tabular}

Columns indicate the type of information used by the classifier; rows indicate which classifier is used. Top two rows are different statistical decomposition techniques of shape parameters (preserving only 1 dimension) and bottom four rows are traditional clinical heart-failure indicators. In the case of ED\&\#8722ES, PCA and IPCA include information on regional wall motion.

\section{Author details}

${ }^{1}$ Auckland Bioengineering Institute, The University of Auckland, Auckland, New Zealand. ${ }^{2} \mathrm{NIH}$ Clinical Center, Bethesda, MD, USA. ${ }^{3}$ Feinberg

Cardiovascular Research Institute, Northwestern University, Chicago, IL, USA.

${ }^{4}$ Donald W. Reynolds Research Institute, Johns Hopkins University, MD, USA.

${ }^{5}$ Diagnostic Cardiovascular Imaging, University California Los Angeles, Los

Angeles, CA, USA.

Published: 30 January 2013

\section{References}

1. Bild DE, et al:. American J. Epidemiology 2002.

2. Kadish $\mathrm{AH}$, et al.. J. CV. Electrophysiology 2009.

3. Fonseca Carissa G, et al:. Bioinformatics 2011.

4. Medrano-Gracia P, et al:. MICCAl-STACOM 2011.

5. Carter KM, et al:. IEEE J. Signal Processing 2009.

\section{doi:10.1186/1532-429X-15-S1-P89}

Cite this article as: Medrano-Gracia et al:: Optimal patient classification via statistical decomposition of a 3D left-ventricular atlas. Journal of

Cardiovascular Magnetic Resonance 2013 15(Suppl 1):P89.

Submit your next manuscript to BioMed Central and take full advantage of:

- Convenient online submission

- Thorough peer review

- No space constraints or color figure charges

- Immediate publication on acceptance

- Inclusion in PubMed, CAS, Scopus and Google Scholar

- Research which is freely available for redistribution

Submit your manuscript at www.biomedcentral.com/submit 